\title{
ADEQUATE SEMIGROUPS
}

\author{
by JOHN FOUNTAIN \\ (Received 20th September 1977)
}

A monoid in which every principal right ideal is projective is called a right PP monoid. Special classes of such monoids have been investigated in (2), (3), (4) and (8). There is a well-known internal characterisation of right PP monoids using the relation $\mathscr{L}^{*}$ which is defined as follows. On a semigroup $S,(a, b) \in \mathscr{L}^{*}$ if and only if the elements $a, b$ of $S$ are related by Green's relation $\mathscr{L}$ in some oversemigroup of $S$. Then a monoid $S$ is a right PP monoid if and only if each $\mathscr{L}^{*}$-class of $S$ contains an idempotent. The existence of an identity element is not relevant for the internal characterisation and in this paper we study some classes of semigroups whose idempotents commute and in which each $\mathscr{L}^{*}$-class contains an idempotent. We call such a semigroup a right adequate semigroup since it contains a sufficient supply of suitable idempotents. Dually we may define the relation $\mathscr{R}^{*}$ on a semigroup and the notion of a left adequate semigroup. A semigroup which is both left and right adequate will be called an adequate semigroup.

Any inverse semigroup is adequate and in investigating adequate semigroups it is natural to look for analogues of results for inverse semigroups. In (6), Howie determines the greatest idempotent separating congruence $\mu$ on an inverse semigroup $S$ and shows that $S / \mu$ is isomorphic to the semilattice of idempotents of $S$ if and only if the idempotents in $S$ are central. In an adequate semigroup there need not be a greatest idempotent separating congruence. However, on an inverse semigroup, $\mu$ is the largest congruence contained in $\mathscr{H}$. Defining $\mathscr{H}^{*}$ to be $\mathscr{L}^{*} \cap \mathscr{R}^{*}$, we determine, in Section 2 , the largest congruence $\mu_{L}$ contained in $\mathscr{L}^{*}$ on a right adequate semigroup and hence find the largest congruence $\mu$ contained in $\mathscr{H}^{*}$ on an adequate semigroup. We also find that the analogue of the second result of Howie mentioned above holds.

It is well known (see (10) or (7, Chapter V)) that if $S$ is an inverse semigroup with semilattice of idempotents $E$, then there is an idempotent separating homomorphism $\theta$ from $S$ into the Munn semigroup $T_{E}$ such that $\theta \circ \theta^{-1}=\mu$ and $\theta$ restricts to an isomorphism between $E$ and the semilattice of idempotents of $T_{E}$. In Section 3, we define a class of right adequate semigroups which we call right type $B$ semigroups. For any semilattice $E$ we define a semigroup $B(E)$ which is right type $B$ and whose semilattice of idempotents $\bar{E}$ is isomorphic to $E$. For any right type $B$ semigroup $S$ with semilattice of idempotents $E$, there is a homomorphism $\theta: S \rightarrow B(E)$ such that $\theta \circ \theta^{-1}=\mu_{L}$ and $\theta$ restricts to an isomorphism between $E$ and $\bar{E}$. In Section 4 we consider adequate semigroups and using $T_{E}$, show that there is an analogue of Munn's result for a class of adequate semigroups which we call type $A$ semigroups. 


\section{Preliminaries}

In this section we give some alternative characterisations of adequate semigroups and some of their elementary properties. We begin by recalling some elementary facts about the relation $\mathscr{L}^{*}$ on a semigroup $S$. It is clear that $\mathscr{L} \subseteq \mathscr{L}^{*}$ and it is well-known that for regular elements $a, b \in S, a \mathscr{L}^{*} b$ if and only if $a \mathscr{L} b$. In particular, if $S$ is a regular semigroup, then $\mathscr{L}=\mathscr{L}^{*}$. From (9) and (11) we have

Lemma 1.1. Let $S$ be a semigroup and let $a, b \in S$. Then the following conditions are equivalent:

(1) $(a, b) \in \mathscr{L}^{*}$;

(2) for all $x, y \in S^{1}, a x=a y$ if and only if $b x=b y$;

(3) there is an $S^{\prime}$-isomorphism $\phi: a S^{\prime} \rightarrow b S^{\prime}$ with $a \phi=b$.

We note that an idempotent $e$ acts as a right identity within its $\mathscr{L}^{*}$-class, for if $(e, a) \in \mathscr{L}^{*}$, then $e^{2}=e 1$ and so by (2), $a e=a 1=a$. From (5), we have

Lemma 1.2. If $U$ is a regular subsemigroup of a semigroup $S$ and $a, b \in U$, then $a, b$ are $\mathscr{L}$-related in $U$ if and only if $a, b$ are $\mathscr{L}$-related in $S$.

Note that in this Lemma, $\mathscr{L}$ may be replaced by $\mathscr{L}^{*}$ since $a, b$ are regular elements.

Proposition 1.3. Let $S$ be a semigroup, $E$ its set of idempotents and $T$ the set of regular elements in $S$. Then the following conditions are equivalent:

(1) $S$ is adequate;

(2) $T$ is an inverse subsemigroup of $S$ and $E$ has non-empty intersection with each $\mathscr{L}^{*}$-class and each $\mathscr{R}^{*}$-class of $S$;

(3) $T$ is an inverse subsemigroup of $S$ and $T$ has non-empty intersection with each $\mathscr{L}^{*}$-class and each $\mathscr{R}^{*}$-class of $S$;

(4) each $\mathscr{L}^{*}$-class and each $\mathscr{R}^{*}$-class of $S$ contains a unique idempotent and the subsemigroup generated by $E$ is regular.

Proof. If (1) holds, then by definition each $\mathscr{L}^{*}$-class and each $\mathscr{R}^{*}$-class of $S$ contains an idempotent. Also, $E$ is a subsemilattice of $S$ and so by $(1$, Section 7.1, Exercise 1), $T$ is an inverse semigroup.

If (2) holds, then since $E \subseteq T$, (3) also holds.

If (3) holds, then $E$ is a semilattice. If $L$ is an $\mathscr{L}^{*}$-class of $S$ and $a \in L \cap T$, then in $T$ and by Lemma 1.2 in $S$ also, $a$ is $\mathscr{L}$-related to $a^{-1} a$ where $a^{-1}$ is the inverse of $a$. Thus each $\mathscr{L}^{*}$-class of $S$ contains an idempotent. Similarly, so does each $\mathscr{R}^{*}$-class of $S$. The uniqueness follows from Lemma 1.2 and the fact that $T$ is inverse.

If (4) holds, then to establish (1), we have only to show that $E$ is a semilattice. Since the subsemigroup $\langle E\rangle$ generated by $E$ is regular, we see from Lemma 1.2 that each $\mathscr{L}$-class and each $\mathscr{R}$-class of $\langle E\rangle$ contains a single idempotent. Thus $\langle E\rangle$ is an inverse semigroup so that $\langle E\rangle=E$.

We now give an example to show that in condition (4) of the above proposition, the assumption that $\boldsymbol{E}$ generates a regular subsemigroup of $S$ cannot be removed. 
Example 1.4.

Let $A=\left(\begin{array}{ll}1 & 0 \\ 0 & 0\end{array}\right), \quad B=\left(\begin{array}{ll}1 & 0 \\ 1 & 0\end{array}\right), \quad C=\left(\begin{array}{ll}1 & 1 \\ 0 & 0\end{array}\right), \quad D=\left(\begin{array}{ll}1 & 1 \\ 1 & 1\end{array}\right)$ and put

$$
S=\left\{2^{m} A, 2^{n} B, 2^{n} C, 2^{n} D: m \geqslant 1, n \geqslant 0\right\} .
$$

It is easy to see that $S$ is a semigroup under matrix multiplication. Further $S$ is generated by $B$ and $C$ and these elements are the only idempotents in $S$. It is routine to check that the $\mathscr{L}^{*}$-classes of $S$ are

$$
\left\{2^{m} A, 2^{n} B: m \geqslant 1, n \geqslant 0\right\}, \quad\left\{2^{n} C, 2^{n} D: n \geqslant 0\right\}
$$

and that the $\mathscr{R}^{*}$-classes are

$$
\left\{2^{m} A, 2^{n} C: m \geqslant 1, n \geqslant 0\right\}, \quad\left\{2^{n} B, 2^{n} D: n \geqslant 0\right\} .
$$

Thus each $\mathscr{L}^{*}$-class and each $\mathscr{R}^{*}$-class contains a unique idempotent but $S$ is not adequate since $B, C$ do not commute.

From the proof of Proposition 1.3 we see that if $S$ is a right adequate semigroup, then each $\mathscr{L}^{*}$-class of $S$ contains a unique idempotent. For an element $a$ of such a semigroup, the idempotent in the $\mathscr{L}^{*}$-class containing $a$ will be denoted by $a^{*}$. In the terminology of (2), $a$ is left $a^{*}$-cancellable. In the dual case of a left adequate semigroup $S, a^{\dagger}$ will denote the idempotent in the $\mathscr{R}^{*}$-class containing the element $a$ of $S$.

A right (left) adequate semigroup $S$ is called a right (left) type $A$ semigroup if $e a=a(e a)^{*}\left(a e=(a e)^{\dagger} a\right)$ for all elements $a$ in $S$ and all idempotents $e$ in $S$. An adequate semigroup $S$ is type $A$ if it is both right and left type $A$. In (4) alternative characterisations of right type $A$ monoids (there called type $A$ monoids) are given. This result does not depend in an essential way on the existence of an identity element and combining it with its left-right dual, we have

Proposition 1.5. Let $S$ be an adequate semigroup. Then the following conditions are equivalent:

(1) $S$ is a type A semigroup;

(2) for every idempotent $e$ in $S$ and every element $a$ in $S, e S^{1} \cap a S^{1}=e a S^{1}$ and $S^{\prime} e \cap S^{\prime} a=S^{\prime} a e$

(3) there are embeddings $\phi_{1}: S \rightarrow S_{1}, \phi_{2}: S \rightarrow S_{2}$ where $S_{1}, S_{2}$ are inverse semigroups and $a^{*} \phi_{1}=\left(a \phi_{1}\right)^{*}=\left(a \phi_{1}\right)^{-1} a \phi_{1}, a^{\dagger} \phi_{2}=\left(a \phi_{2}\right)^{\dagger}=a \phi_{2}\left(a \phi_{2}\right)^{-1}$.

We shall obtain further characterisations of type $A$ semigroups in Section 4 . We conclude this section by listing some elementary properties of adequate semigroups which we shall subsequently use without further mention. then

Proposition 1.6. If $S$ is an adequate semigroup with semilattice of idempotents $E$,

(1) for all $a, b \in S, a \mathscr{R}^{*} b$ if and only if $a^{\dagger}=b^{\dagger} ; a \mathscr{L}^{*} b$ if and only if $a^{*}=b^{*}$;

(2) for all $a, b \in S,(a b)^{*}=\left(a^{*} b\right)^{*}$ and $(a b)^{\dagger}=\left(a b^{\dagger}\right)^{\dagger}$;

(3) for all $a, b \in S,(a b)^{*} \leqslant b^{*}$ and $(a b)^{\dagger} \leqslant a^{\dagger}$ where $\leqslant$ is the usual ordering on $E$. 
Proof. (1) follows immediately from Proposition 1.3 and (2) follows from (1) and the fact that $\mathscr{L}^{*}\left(\mathscr{R}^{*}\right)$ is a right (left) congruence on $S$. (3) is trivial.

We note that if $e \in E$, then from (2) we have $(a e)^{*}=a^{*} e$ and $(e a)^{\dagger}=e a^{\dagger}$. Of course, the appropriate parts of the above result hold if the semigroup $S$ is simply left or right adequate.

\section{The congruence $\mu$}

In this section we give a characterisation of the largest congruence $\mu_{L}$ contained in $\mathscr{L}^{*}$ on a right adequate semigroup and by combining this with its left-right dual, we obtain a description of the largest congruence $\mu$ contained in $\mathscr{H}^{*}$ on an adequate semigroup. We then examine for the appropriate type of semigroup $S$, when $S / \mu_{L} \approx E$ and when $S / \mu \approx E$. In the adequate case we find that $S / \mu \approx E$ precisely when $E$ is central in $S$.

Let $S$ be a right adequate semigroup with semilattice of idempotents $E$. For each element $a$ of $S$, define the mapping $\alpha_{a}: E^{1} \rightarrow E^{1}$ by $x \alpha_{a}=(x a)^{*}$.

Lemma 2.1. (1) The mapping $\alpha_{a}$ is isotone.

(2) The mapping $\alpha: S \rightarrow \mathscr{T}\left(E^{1}\right)$ by a $\alpha=\alpha_{a}$ is a homomorphism.

(3) If $S$ is right type $A$, then $\alpha_{a}$ is a homomorphism.

Proof. (1) Let $x, y \in E^{1}$ and $a \in S$. Then

$$
x y a(y a)^{*}(x a)^{*}=x y a(x a)^{*}=y x a(x a)^{*}=y x a=x y a
$$

and hence

$$
(x y a)^{*}=\left(x y a(y a)^{*}(x a)^{*}\right)^{*}=(x y a)^{*}(y a)^{*}(x a)^{*} .
$$

Thus if $x \leqslant y$, then $x y=x$ so that $(x a)^{*}=(x a)^{*}(y a)^{*}$, that is, $(x a)^{*} \leqslant(y a)^{*}$.

(2) If $x \in E^{1}$ and $a, b \in S$, then

$$
x \alpha_{a} \alpha_{b}=\left((x a)^{*} b\right)^{*}=(x a b)^{*}=x \alpha_{a b}
$$

so that $\alpha_{a} \alpha_{b}=\alpha_{a b}$ as required.

(3) If $S$ is right type $A$, then for $x, y \in E^{1}$ we have

$$
(x y) \alpha_{a}=(x y a)^{*}=\left(x a(y a)^{*}\right)^{*}=(x a)^{*}(y a)^{*}=x \alpha_{a} y \alpha_{a} .
$$

For a general right adequate semigroup $S$ and element $a$ of $S$, the mapping $\alpha_{a}$ need not be a homomorphism as the following example demonstrates. is:-

Example 2.2. Let $S$ be the eight element semigroup whose multiplication table 


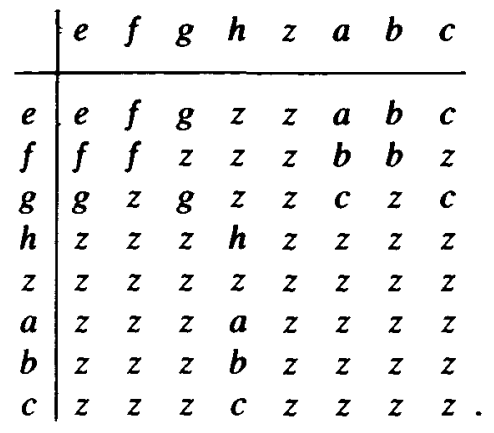

Associativity may be checked directly or by observing that $E=\{e, f, g, h, z\}$ is a semilattice and that $S$ may be identified with the subsemigroup of the semigroup of all partial mappings of $E$ generated by $\{a\} \cup\left\{I_{x}: x \in E\right\}$ where $I_{x}$ is the identity map of $x E$ and $a:\{e, f, g, z\} \rightarrow\{h, z\}$ by $z a=z$ and $x a=h$ for $x \neq z$. From the table one sees that the $\mathscr{L}^{*}$-classes of $S$ are $\{h, a, b, c\},\{e\},\{f\},\{g\},\{z\}$ and the $\mathscr{R}^{*}$-classes of $S$ are $\{e, a\},\{f, b\}$, $\{g, c\},\{h\},\{z\}$. Thus $S$ is an adequate semigroup. The mapping $\alpha_{a}$ is not a homomorphism even if its domain is restricted to $a^{\dagger} E=e E$ since $(f a)^{*}(g a)^{*}=b^{*} c^{*}=h$ whereas $(f g a)^{*}=(z a)^{*}=z$.

We can now determine the largest congruence contained in $\mathscr{L}^{*}$ on a right adequate semigroup.

Proposition 2.3. Let $S$ be a right adequate semigroup with semilattice of idempotents $E$. Then the largest congruence $\mu_{L}$ contained in $\mathscr{L}^{*}$ is given by

$$
\begin{aligned}
\mu_{L} & =\left\{(a, b) \in S \times S: \alpha_{a}=\alpha_{b}\right\} \\
& =\left\{(a, b) \in S \times S:(x a)^{*}=(x b)^{*} \text { for all } x \in E^{1}\right\} .
\end{aligned}
$$

Proof. That $\mu_{L}$ is a congruence is immediate from (2) of Lemma 2.1. If $(a, b) \in \mu_{L}$, then $a^{*}=b^{*}$ so that $(a, b) \in \mathscr{L}^{*}$, that is, $\mu_{L} \subseteq \mathscr{L}^{*}$. Let $\nu$ be a congruence on $S$ with $\nu \subseteq \mathscr{L}^{*}$. If $(a, b) \in \nu$, then for any $x \in E^{1},(x a, x b) \in \nu$ so that $(x a, x b) \in \mathscr{L}^{*}$, that is, $(x a)^{*}=(x b)^{*}$ which gives $(a, b) \in \mu_{L}$ and $\nu \subseteq \mu_{L}$.

Of course, the dual result shows that the largest congruence $\mu_{R}$ contained in $\mathscr{R}^{*}$ on a left adequate semigroup $S$ with semilattice of idempotents $E$ is given by

$$
\begin{aligned}
\mu_{R} & =\left\{(a, b) \in S \times S:(a x)^{\dagger}=(b x)^{\dagger} \text { for all } x \in E^{\prime}\right\} \\
& =\left\{(a, b) \in S \times S: \beta_{a}=\beta_{b}\right\}
\end{aligned}
$$

where $\beta_{a}: E^{1} \rightarrow E^{1}$ by $x \beta_{a}=(a x)^{\dagger}$. If $S$ is an adequate semigroup, we may combine the expressions for $\mu_{L}$ and $\mu_{R}$ to obtain an expression for $\mu=\mu_{L} \cap \mu_{R}$, the largest congruence contained in $\mathscr{H}^{*}$. For such a semigroup with semilattice of idempotents $E$ we can make a slight modification in the expression for $\mu$ by noting that if $a, b \in S$ with $a^{\dagger}=b^{\dagger}$ and $(x a)^{*}=(x b)^{*}$ for all $x \in a^{\dagger} E$, then for any $y \in E$, we have $(y a)^{*}=$ $\left(y a^{\dagger} b\right)^{*}=(y b)^{*}$, that is, $(a, b) \in \mu_{L}$. Thus

$$
\begin{aligned}
\mu & =\left\{(a, b) \in S \times S: a^{\dagger}=b^{\dagger}, a^{*}=b^{*} \text { and }(x a)^{*}=(x b)^{*},(a y)^{\dagger}=(b y)^{\dagger}\right. \\
& \text { for all } \left.x \in a^{\dagger} E \text { and for all } y \in a^{*} E\right\} \\
& =\left\{(a, b) \in S \times S: \alpha_{a}=\alpha_{b} \text { and } \beta_{a}=\beta_{b}\right\}
\end{aligned}
$$


where we interpret $\alpha_{a}$ to be the mapping $\alpha_{a}: a^{\dagger} E \rightarrow a^{*} E$ by $x \alpha_{a}=(x a)^{*}$ and $\beta_{a}: a^{*} E \rightarrow$ $a^{\dagger} E$ by $y \beta_{a}=(a y)^{\dagger}$.

The classes of adequate semigroups and right adequate semigroups are not closed under homomorphic images. We are thus led to consider when $S$ is right adequate, whether or not $S / \mu_{L}$ is right adequate and when $S$ is adequate whether or not $S / \mu$ is adequate. We show that this is not necessarily the case in the following example of an adequate semigroup $S$ which is right type $A$ and satisfies $\mu_{L}=\mu$ but is such that $S / \mu$ is not right adequate.

Example 2.4. Let $A$ be the infinite cyclic semigroup with generator $a$ and let $B$ be the infinite cyclic monoid with generator $b$ and identity $e$. Let $S=A \cup B \cup\{1\}$ and define a product on $S$ which extends those on $A$ and $B$ and has 1 as the identity by putting $a^{m} b^{n}=b^{m+n}, b^{n} a^{m}=a^{n+m}$ for integers $m>0$ and $n \geqslant 0$ where $b^{0}=e$.

It is routine to check that $S$ is a monoid with idempotents $1, e$. The $\mathscr{L}^{*}$-classes of $S$ are $A \cup\{1\}, B$ and the $\mathscr{R}^{*}$-classes are $\{1\}, A \cup B$. Thus $S$ is an adequate monoid. Further, it is easily seen that $x s=s(x s)^{*}$ for all idempotents $x$ in $S$ and all elements $s$ in $S$ so that $S$ is right type $A$. Finally it is not difficult to check that the $\mu_{L}$-classes of $S$ are $\{1\}, A, B$ and that $\mu_{R}=\mathscr{R}^{*}$. Thus $\mu_{L} \subseteq \mu_{R}$ so that $\mu_{L}=\mu$ and $S / \mu \approx R_{2} \cup\{1\}$ where $R_{2}$ is the two element right zero semigroup. Since the idempotents of $S / \mu$ do not commute, $S / \mu$ is not a right adequate semigroup.

We now consider under what conditions on a right adequate semigroup $S$, we have $S / \mu_{L} \approx E$. We first need the following lemma.

Lemma 2.5. Let $S$ be a right adequate semigroup with semilattice of idempotents $E$. If the idempotents of $S / \mu_{L}$ commute, then $\bar{E}=\left\{e \mu_{L}: e \in E\right\}$ is the set of idempotents of $S / \mu_{L}$ and $\theta: E \rightarrow \bar{E}$ by $e \theta=e \mu_{L}$ is an isomorphism.

Proof. Certainly all the elements of $\bar{E}$ are idempotents. If $a \mu_{L}$ is an idempotent of $S / \mu_{L}$, then for all $e \in E,(a e, e a) \in \mu_{L}$ so that $(a e, e a) \in \mathscr{L}^{*}$ and $(e a)^{*}=(a e)^{*}=a^{*} e$. Thus $(x a)^{*}=\left(x a^{*}\right)^{*}$ for all $x \in E^{1}$, that is, $\left(a, a^{*}\right) \in \mu_{L}$ and $a \mu_{L}=a^{*} \mu_{L} \in \bar{E}$.

Clearly $\theta$ is a surjective homomorphism. It is also injective; for if $(e, f) \in$ $(E \times E) \cap \mu_{L}$, then $(e, f) \in \mathscr{L}^{*}$ so that $e=f$.

Corollary 2.6. Let $S$ be an adequate semigroup with semilattice of idempotents $E$. If the idempotents of $S / \mu$ commute, then $\bar{E}=\{e \mu: e \in E\}$ is the set of idempotents of $S / \mu$ and $\theta: E \rightarrow \bar{E}$ by $e \theta=e \mu$ is an isomorphism.

Proof. Certainly $\bar{E}$ consists of idempotents. If $a \mu$ is an idempotent of $S / \mu$, then $(a e, e a) \in \mu$ for all $e \in E$. In particular, $\left(a a^{\dagger}, a\right) \in \mu$ so that $\left(a a^{\dagger}, a\right) \in \mathscr{L}^{*}$ and $a^{*}=$ $\left(a a^{\dagger}\right)^{*}=a^{*} a^{\dagger}$. Similarly, since $\left(a, a^{*} a\right) \in \mu$, we get $a^{\dagger}=a^{*} a^{\dagger}$ and so $a^{\dagger}=a^{*}$. Since $\mu \subseteq \mu_{L}$, we see from the proof of the Lemma that $\left(a, a^{*}\right) \in \mu_{L}$ and dually $\left(a, a^{\dagger}\right) \in \mu_{R}$. Hence $\left(a, a^{*}\right) \in \mu$ and $a \mu \in \bar{E}$.

That $\theta$ is an isomorphism is clear. 
Proposition 2.7. Let $S$ be a right adequate semigroup with semilattice of idempotents $E$. Then the following conditions are equivalent:

(1) $S / \mu_{L} \approx E$

(2) $\mu_{L}=\mathscr{L}^{*}$;

(3) $(a e, e a) \in \mathscr{L}^{*}$ for all $e \in E$ and all $a \in S$.

Proof. It follows from Lemma 2.5 that $S / \mu_{L} \approx E$ if and only if each $\mu_{L}$-class contains an idempotent. Since $\mu_{L} \subseteq \mathscr{L}^{*}$ and each $\mathscr{L}^{*}$-class contains a unique idempotent, we see that this is the case if and only if $\mu_{L}=\mathscr{L}^{*}$.

Suppose that $\mu_{L}=\mathscr{L}^{*}$. Then for each element $a$ in $S$ we have $\left(a^{*}, a\right) \in \mu_{L}$, that is, $x a^{*}=(x a)^{*}$ for all $x \in E^{1}$. Since $(a x)^{*}=a^{*} x=x a^{*}$, we see that for all $e \in E$ we have $(a e, e a) \in \mathscr{L}^{*}$. Reversing the argument shows that if condition (3) holds, then $\mu_{L}=\mathscr{L}^{*}$.

In the case when $S$ is a right type $A$ semigroup we can deduce a stronger result. Recall that $S=\cup_{\alpha \in Y} S_{\alpha}$ is a strong semilattice of the semigroups $S_{\alpha}$ when $Y$ is a semilattice, $\left\{S_{\alpha}: \alpha \in Y\right\}$ is a disjoint family of semigroups and for $\alpha, \beta \in Y$ with $\beta \leqslant \alpha$ there are homomorphisms $\phi_{\alpha, \beta}: S_{\alpha} \rightarrow S_{\beta}$ satisfying

(1) $\phi_{\alpha, a}$ is the identity map for each $\alpha \in Y$;

(2) $\phi_{\alpha, \beta} \phi_{\beta, \gamma}=\phi_{\alpha, \gamma}$ for every $\alpha, \beta, \gamma$ in $Y$ such that $\alpha \geqslant \beta \geqslant \gamma$;

and such that the product in $S$ is given by $a b=a \phi_{\alpha, \alpha \beta} b \phi_{\beta, \alpha \beta}$ where $a \in S_{\alpha}, b \in S_{\beta}$.

Now we have

Corollary 2.8. Let $S$ be a right type A semigroup with semilattice of idempotents $E$. Then the following conditions are equivalent:

(1) $S / \mu_{L} \approx E$;

(2) $E$ is central in $S$;

(3) $S$ is a strong semilattice of left cancellative monoids.

Proof. The equivalence of (2) and (3) follows from (3). From Proposition 2.7 we see that if $E$ is central in $S$, then $S / \mu_{L} \approx E$. Finally, if condition (1) holds, then by Proposition 2.7, (ae,ea) $\in \mathscr{L}^{*}$ for all $e \in E$ and all $a \in S$. Hence, since $S$ is right ty pe $A$, we have $e a=a(e a)^{*}=a(a e)^{*}=a a^{*} e=a e$, that is, $E$ is central in $S$.

We conclude this section by giving conditions on an adequate semigroup $S$ which are equivalent to $S / \mu \approx E$.

Proposition 2.9. Let $S$ be an adequate semigroup with semilattice of idempotents $E$. Then the following conditions are equivalent:

(1) $S / \mu \approx E$;

(2) for all elements $a$ of $S, a^{*}=a^{\dagger}$;

(3) $\mathscr{L}^{*}=\mathscr{H}^{*}=\mathscr{R}^{*}$;

(4) each $\mathscr{H}^{*}$-class of $S$ contains an idempotent;

(5) $E$ is central in $S$;

(6) $S$ is a strong semilattice of cancellative monoids.

Proof. Suppose that $S / \mu \approx E$. Then for any element $a$ of $S$, we have $\left(a a^{*}, a^{*} a\right) \in \mu$ and since $\mu \subseteq \mathscr{R}^{*}$, this gives $\left(a^{*} a\right)^{\dagger}=a^{\dagger}$, that is, $a^{*} a^{\dagger}=a^{\dagger}$. Similarly, $\left(a a^{\dagger}, a^{\dagger} a\right) \in \mu$ and we get $a^{*} a^{\dagger}=a^{\dagger}$. 
Suppose that $a^{*}=a^{\dagger}$ for all elements $a$ in $S$. Then for $a, b \in S$ we have $(a, b) \in \mathscr{L}^{*}$ if and only if $a^{*}=b^{*}$, that is, $a^{\dagger}=b^{\dagger}$, which is true if and only if $(a, b) \in \mathscr{R}^{*}$. Thus $\mathscr{L}^{*}=\mathscr{R}^{*}=\mathscr{H}^{*}$.

It î clear that condition (3) implies condition (4) and that if condition (4) holds, then so does condition (2). Thus the first four conditions are equivalent.

Now suppose that $a^{*}=a^{\dagger}$ for all elements $a$ in $S$. Then for $a \in S, e \in E$ we have

$$
a e=(a e)^{\dagger} a e=(a e)^{*} a e=a^{*} e a e=e a^{*} a e=e a e
$$

and similarly $e a=e a e$, so that $e a=a e$ and $E$ is central in $S$.

If $E$ is central in $S$, then it follows from the results of (3) that condition (6) holds. Finally, it is easy to see that condition (6) implies condition (2).

\section{Right fundamental semigroups}

We shall say that a right adequate semigroup $S$ is right fundamental if $\mu_{L}=\iota$. The reader may verify that Example 2.2 is right fundamental. We begin this section by showing that if $S$ and $S / \mu_{L}$ are right adequate, then $S / \mu_{L}$ is right fundamental. We then introduce a class of right adequate semigroups called right type $B$ semigroups and show that if $S$ is right type $B$, then so is $S / \mu_{L}$. For any semilattice $E$ we define a semigroup $B(E)$ which is both right type $B$ and right type $A$. Further, any right type $B$ semigroup which is right fundamental and has semilattice of idempotents $E$ is isomorphic to a full subsemigroup of $B(E)$. By a full subsemigroup we mean one which contains all the idempotents of the containing semigroup.

Lemma 3.1. Let $S$ be a right adequate semigroup. If $S / \mu_{L}$ is right adequate, then $\left(a \mu_{L}\right)^{*}=a^{*} \mu_{L}$ for any element $a$ in $S$.

Proof. By Lemma 2.5 we have $\left(a \mu_{L}\right)^{*}=e \mu_{L}$ for some idempotent $e$ in $S$. Since $a \mu_{L} a^{*} \mu_{L}=a \mu_{L}=a \mu_{L} e \mu_{L}$ we have $e \mu_{L} a^{*} \mu_{L}=e \mu_{L}$, that is, $\left(e a^{*}, e\right) \in \mu_{L}$ and consequently $e a^{*}=e$. On the other hand, $(a, a e) \in \mu_{L}$ gives $a^{*}=(a e)^{*}=a^{*} e$ so that $e=a^{*}$.

Proposition 3.2. Let $S$ be a right adequate semigroup with semilattice of idempotents $E$. If $S / \mu_{L}$ is right adequate, then $S / \mu_{L}$ is right fundamental.

Proof. By Lemma 2.5, every idempotent in $S / \mu_{L}$ has the form $e \mu_{L}$ where $e \in E$. Thus we have to show that if $a, b \in S$ and $\left(x \mu_{L} a \mu_{L}\right)^{*}=\left(x \mu_{L} b \mu_{L}\right)^{*}$ for all $x \in E^{1}$, then $a \mu_{L}=b \mu_{L}$. By Lemma $3.1\left(x a \mu_{L}\right)^{*}=\left(x b \mu_{L}\right)^{*}$ gives $\left((x a)^{*},(x b)^{*}\right) \in \mu_{L}$. Since $\mu_{L}$ is idempotent separating, being contained in $\mathscr{L}^{*}$, we have $(x a)^{*}=(x b)^{*}$ for all $x \in E^{1}$, that is, $a \mu_{L}=b \mu_{L}$.

We now define a right type $B$ semigroup to be a right adequate semigroup $S$ with semilattice of idempotents $E$ which satisfies:

(1) for all $x, y$ in $E^{1}$ and all elements $a$ in $S,(x y a)^{*}=(x a)^{*}(y a)^{*}$;

(2) for all elements $a$ in $S$ and all idempotents $e \leqslant a^{*}$, there is an element $x$ in $E^{1}$ such that $e=(x a)^{*}$. 
Note that condition (1) asserts that the mapping $\alpha_{a}: E^{1} \rightarrow E^{1}$ is a homomorphism while condition (2) asserts that $\operatorname{Im} \alpha_{a}=a^{*} E$. We have seen in Lemma 2.1 that right type $A$ semigroups satisfy condition (1). However, not all right type $A$ semigroups are right type $B$, for in Example 2.4 we have $a^{*}=1$ and $(e a)^{*}=a^{*}=1$ so that $e \in a^{*} E$ but $e \notin \operatorname{Im} \alpha_{a}$. On the other hand, not all right type $B$ semigroups are right type $A$ as the following example demonstrates.

Example 3.3. Let $\mathbf{N}$ denote the set of natural numbers and put $I=\mathbf{N} \times \mathbf{N}$. On $S=\mathrm{N} \cup I$ define an operation ${ }^{\circ}$ as follows:

$$
\begin{aligned}
\text { for } m, n, h, k & \in \mathrm{N}, \\
m \circ n & =m+n \\
m \circ(h, k) & =(m+h, k) \\
(h, k) \circ m & =(h, k+m) \\
(h, k) \circ(m, n) & =(h, k+m+n) .
\end{aligned}
$$

It is readily verified that $\circ$ is associative, that the idempotents of $S$ are $0,(0,0)$ and that $I$ is an ideal of $S$. It is not difficult to check that the $\mathscr{L}^{*}$-classes of $S$ are $\mathbf{N}$ and $I$ so that $S$ is right adequate. Since $(0,0) \circ k=(0, k)$ whereas $k \circ((0,0) \circ k)^{*}=k \circ(0,0)=(k, 0)$, we see that $S$ is not right type $A$. However, since the set of idempotents $E=\{0,(0,0)\}$ is a chain with identity 0 , the isotone map $\alpha_{a}: E \rightarrow E$ must be a homomorphism for any $a \in S$. If $a \in I$, then $a^{*}=(0,0)$ so that $\operatorname{Im} \alpha_{a}=\{(0,0)\}=a^{*} \circ E$ and if $a \in \mathrm{N}$, then $a^{*}=0$ and $((0,0) \circ a)^{*}=(0,0)$ so that $\operatorname{Im} \alpha_{a}=E=a^{*} \circ E$. Thus $S$ is a right type $B$ semigroup.

Now let $E$ be a semilattice and define $B(E)$ to be the subset of $\mathscr{T}\left(E^{1}\right)$ consisting of those mappings $\theta$ which satisfy:

(A) $\theta$ is a homomorphism;

(B) $\operatorname{Im} \theta=(1 \theta) E$.

Proposition 3.4. $B(E)$ is a subsemigroup of $\mathscr{T}\left(E^{1}\right)$ and the idempotents of $B(E)$ form a semilattice isomorphic to $E . B(E)$ is right type $B$, right type $A$ and right fundamental.

Proof. If $\theta, \psi \in B(E)$, it is clear that defining condition (A) is satisfied by $\theta \psi$. Now $1 \theta \psi=1 \theta \psi 1 \psi$ so that if $x \in(1 \theta \psi) E$, then $x=(1 \theta \psi)(1 \psi) x$ and $(1 \psi) x=y \psi$ for some $y \in E^{1}$ since $\operatorname{Im} \psi=(1 \psi) E$. Thus $x=(1 \theta \psi)(y \psi)=((1 \theta) y) \psi$. Since $\operatorname{Im} \theta=(1 \theta) E$, we have $(1 \theta) y=z \theta$ for some $z \in E^{1}$ so that $x=z \theta \psi$ and $(1 \theta \psi) E \subseteq \operatorname{Im} \theta \psi$. The opposite inclusion is clear and hence $\theta \psi \in B(E)$.

Suppose that $\theta$ is an idempotent in $B(E)$ and let $1 \theta=e$. Then $e \theta=1 \theta^{2}=1 \theta=e$. If $x \in E^{\prime}$, then $x e \in e E$ so that $x e=y \theta$ for some $y \in E^{1}$ and consequently $x \theta=(x \theta) e=$ $x \theta e \theta=(x e) \theta=y \theta^{2}=y \theta=x e$. Thus $\theta=\lambda_{e}$ where $\lambda_{e}: E^{1} \rightarrow E^{1}$ by $x \lambda_{e}=x e$. Clearly the map sending $e$ to $\lambda_{e}$ is an isomorphism between $E$ and the set $\tilde{E}$ of idempotents of $B(E)$.

Now let $\theta$ be any element of $B(E)$ and let $1 \theta=e$. Clearly $\theta \lambda_{e}=\theta$. Also for any $x \in E^{1}, e x=y \theta$ for some $y \in E^{\prime}$, so that if $\phi, \psi \in B(E)$ and $\theta \phi=\theta \psi$, then we have 


$$
x \lambda_{e} \phi=(e x) \phi=y \theta \phi=y \theta \psi=(e x) \psi=x \lambda_{e} \psi,
$$

so that $\lambda_{e} \phi=\lambda_{e} \psi$. Hence $\left(\lambda_{e}, \theta\right) \in \mathscr{L}^{*}$ and $B(E)$ is right adequate.

To see that $B(E)$ is right type $A$, let $\theta \in B(E), e \in E$. Then for any $x \in E^{\prime}$, we have $x \lambda_{e} \theta=(x e) \theta=x \theta e \theta=x \theta \lambda_{e \theta}$ so that $\lambda_{e} \theta=\theta \lambda_{e \theta}=\theta\left(\lambda_{e} \theta\right)^{*}$.

Since $B(E)$ is right type $A$, to show that it is also right type $B$, we need only show that $\theta^{*} \tilde{E} \subseteq \operatorname{Im} \alpha_{\theta}$ for $\theta \in B(E)$. Now $\theta^{*}=\lambda_{e}$ where $e=1 \theta$ and if $\lambda_{f} \in \tilde{E}$ with $\lambda_{f} \leqslant \lambda_{e}$, then $f \in E$ and $f \leqslant e$ so that $f \in \operatorname{Im} \theta$. If $f=x \theta, x \in E^{1}$, then $\lambda_{f}=\lambda_{x \theta}=\left(\lambda_{x} \theta\right)^{*}=\lambda_{x} \alpha_{\theta}$ and hence $B(E)$ is right type $B$.

Finally, if $\theta, \psi \in B(E)$ and $(\theta, \psi) \in \mu_{L}$, then for all $x \in E^{1}\left(\lambda_{x} \theta\right)^{*}=\left(\lambda_{x} \psi\right)^{*}$, that is, $\lambda_{x \theta}=\lambda_{x \psi}$ so that $x \theta=x \psi$ and $\theta=\psi$. Thus $B(E)$ is right fundamental.

We next record the fact that full subsemigroups of a semigroup $S$ inherit certain properties of $S$. The easy proof is left to the reader.

Proposition 3.5. Let $S$ be a semigroup and $T$ be a full subsemigroup of $S$. Then

(1) if $S$ is right adequate, then so is $T$;

(2) if $S$ is right type $A$, then so is $T$;

(3) if $S$ is right type $B$, then so is $T$;

(4) if $S$ is right adequate and right fundamental, then so is $T$.

We can now easily obtain the main results of this section.

Proposition 3.6. Let $S$ be a right type $B$ semigroup with semilattice of idempotents E. Then $S / \mu_{L}$ is isomorphic to a full subsemigroup of $B(E)$.

Proof. By Lemma 2.1, $\alpha: S \rightarrow \mathscr{T}\left(E^{\prime}\right)$ by $a \alpha=\alpha_{a}$ is a homomorphism and $S / \mu_{L} \approx$ $\operatorname{Im} \alpha$. Since $S$ is right type $B$, we have that $\alpha_{a}$ is a homomorphism and $\operatorname{Im} \alpha_{a}=a^{*} E=$ $\left(1 \alpha_{a}\right) E$. Thus $\operatorname{Im} \alpha \subseteq B(E)$. It is easy to see that for each $e \in E, \alpha_{e}=\lambda_{e}$ so that $\operatorname{Im} \alpha$ is a full subsemigroup of $B(E)$.

The following corollaries are now immediate from Propositions 3.2, 3.4, 3.5 and 3.6 .

Corollary 3.7. If $S$ is a right type $B$ semigroup, then $S / \mu_{L}$ is right type A, right type $B$ and right fundamental.

Corollary 3.8. A right type $B$ semigroup $S$ with semilattice of idempotents $E$ is right fundamental if and only if it is isomorphic to a full subsemigroup of $B(E)$. A.

Corollary 3.9. A right type B semigroup which is right fundamental is right type

\section{Fundamental adequate semigroups}

We shall say that an adequate semigroup is fundamental if $\mu=\imath$. The semigroup of Example 2.2 is fundamental. We begin by noting the two-sided analogues of Lemma 
3.1 and Proposition 3.2. We omit the proofs as these are similar to those of the results mentioned using Corollary 2.6 instead of Lemma 2.5 .

Lemma 4.1. Let $S$ be an adequate semigroup. If $S / \mu$ is adequate, then $(a \mu)^{*}=a^{*} \mu$ and $(a \mu)^{\dagger}=a^{\dagger} \mu$ for all elements $a$ of $S$.

Proposition 4.2. Let $S$ be an adequate semigroup. If $S / \mu$ is an adequate semigroup, then $S / \mu$ is fundamental.

As noted in Section 2, when $S$ is an adequate semigroup with semilattice of idempotents $E$ and $a \in S$, the domains of the mappings $\alpha_{a}$ and $\beta_{a}$ can be usefully restricted to $a^{\dagger} E$ and $a^{*} E$ respectively. Henceforth when we use $\alpha_{a}$ or $\beta_{a}$ we shall mean $\alpha_{a}: a^{\dagger} E \rightarrow a^{*} E$ by $e \alpha_{a}=(e a)^{*}$ and $\beta_{a}: a^{*} E \rightarrow a^{\dagger} E$ by $e \beta_{a}=(a e)^{\dagger}$. In order to use $\alpha_{a}, \beta_{a}$ to obtain a representation of $S$ on a full subsemigroup of an adequate semigroup of mappings it seems essential to impose the condition that $\alpha_{a}$ and $\beta_{a}$ are both injective for each $a$ in $S$. As we shall see, this is equivalent to the condition that $S$ be a type $A$ semigroup and in this case the appropriate semigroup of mappings is the Munn semigroup $T_{E}$. We recall that for a semilattice $E, T_{E}$ consists of all isomorphisms between principal ideals of $E$ and refer the reader to (7, Chapter V) for further information about $T_{E}$ including the proof that $T_{E}$ is an inverse semigroup with semilattice of idempotents isomorphic to $E$.

Lemma 4.3. If $a$ is an element of an adequate semigroup $S$, then $x \alpha_{a} \beta_{a} \geqslant x$ for all $x \in a^{\dagger} E$ and $y \beta_{a} \alpha_{a} \geqslant y$ for all $y \in a^{*} E$.

Proof. If $x \in a^{\dagger} E$, then $x \alpha_{a} \beta_{a}=\left(a(x a)^{*}\right)^{\dagger}$. Since $x\left(a(x a)^{*}\right)^{\dagger}=\left(x a(x a)^{*}\right)^{\dagger}=$ $(x a)^{\dagger}=x a^{\dagger}=x$, we have $x \leqslant x \alpha_{a} \beta_{a}$. Similarly, for $y \in a^{*} E$ we have $y \leqslant y \beta_{a} \alpha_{a}$.

Proposition 4.4. For an adequate semigroup $S$ with semilattice of idempotents $E$ the following conditions are equivalent:

(1) $S$ is a type A semigroup;

(2) for each element a of $S$, the mappings $\alpha_{a}$ and $\beta_{a}$ are injective;

(3) for each element $a$ of $S$, the mappings $\alpha_{a}$ and $\beta_{a}$ are inverse isomorphisms.

Proof. Suppose that $S$ is a type $A$ semigroup and let $a \in S, e, f \in a^{\dagger} E$. If $e \alpha_{a}=f \alpha_{a}$, then $(e a)^{*}=(f a)^{*}$ so that $e a=a(e a)^{*}=a(f a)^{*}=f a$. Hence $e=e a^{\dagger}=(e a)^{\dagger}=(f a)^{\dagger}=$ $f a^{\dagger}=f$ and $\alpha_{a}$ is injective. Similarly, $\beta_{a}$ is injective.

Suppose now that $\alpha_{a}, \beta_{a}$ are injective for each $a \in S$. Let $x \in a^{\dagger} E$ and let $e=x \alpha_{a} \beta_{a}$. Certainly $e \in a^{\dagger} E$ and by Lemma $4.3, x \leqslant e$. Since $(e a)^{\dagger}=e a^{\dagger}=e$, the domain of $\alpha_{e a}$ is $e E$. Also $\alpha_{e a}=\alpha_{e} \alpha_{a} \mid e E$ and $\beta_{e a}=\beta_{a} \beta_{e} \mid(e a)^{*} E$ so that

$$
x \alpha_{e a} \beta_{e a}=x \alpha_{e} \alpha_{a} \beta_{a} \beta_{e}=x \alpha_{a} \beta_{a} \beta_{e}=e \beta_{e}=e
$$

and

$$
e \alpha_{e a} \beta_{e a}=e \alpha_{e} \alpha_{a} \beta_{a} \beta_{e}=\left(e \alpha_{a} \beta_{a}\right) e=e
$$

since $e \leqslant e \alpha_{a} \beta_{a}$ by Lemma 4.3. Since $\alpha_{e a}, \beta_{e a}$ are injective, we have $e=x$. Hence $\alpha_{a} \beta_{a}$ 
is the identity map on $a^{\dagger} E$. A similar argument shows that $\beta_{a} \alpha_{a}$ is the identity map on $a^{*} E$. Since $\alpha_{a}, \beta_{a}$ are isotone, they are thus inverse order-isomorphisms and hence semigroup isomorphisms.

Suppose that condition (3) holds for $S$ and let $a \in S, e \in E$. If $x=e a^{\dagger}$, then $\left(a(x a)^{*}\right)^{\dagger}=x \alpha_{a} \beta_{a}=x$ and so $e a=x a=x a(x a)^{*}=a(x a)^{*}=a(e a)^{*}$. Similarly $a e=$ $(a e)^{\dagger} a$ and so $S$ is a type $A$ semigroup.

Since $\alpha_{a}: a^{\dagger} E \rightarrow a^{*} E$ is an isomorphism when $S$ is a type $A$ semigroup with semilattice of idempotents $E$, we have $\alpha_{a} \in T_{E}$ and we can define a mapping $\theta: S \rightarrow T_{E}$ by $a \theta=\alpha_{a}$. We then have

Proposition 4.5. Let $S$ be a type A semigroup with semilattice of idempotents $E$. Then the mapping $\theta$ is a homomorphism onto a full subsemigroup of $T_{E}$ and $\theta \circ \theta^{-1}=\mu$.

Proof. If $a, b \in S$, then $\alpha_{a}: a \dagger E \rightarrow a^{*} E$ and $\alpha_{b}: b \dagger E \rightarrow b^{*} E$ so that $\alpha_{a} \alpha_{b}$ has domain $\quad\left(a^{*} E \cap b^{\dagger} E\right) \alpha_{a}^{-1}=\left(a^{*} b^{\dagger} E\right) \beta_{a}=\left(a^{*} b^{\dagger}\right) \beta_{a} E=\left(a a^{*} b^{\dagger}\right)^{\dagger} E=\left(a b^{\dagger}\right)^{\dagger} E=(a b)^{\dagger} E$. Now $\alpha_{a b}$ has domain $(a b)^{\dagger} E$ and for $x \in(a b)^{\dagger} E, x \alpha_{a b}=x \alpha_{a} \alpha_{b}$ so that $\alpha_{a b}=\alpha_{a} \alpha_{b}$ in $T_{E}$ and $\theta$ is a homomorphism.

The idempotents of $T_{E}$ have the form $I_{e E}$ and since $e \theta=\alpha_{e}=I_{e E}$ we see that $\operatorname{Im} \theta$ is a full subsemigroup of $T_{E}$.

Finally, for $a, b \in S$ we have $(a, b) \in \mu$ if and only if $\alpha_{a}=\alpha_{b}$ and $\beta_{a}=\beta_{b}$ which is true if and only if $\alpha_{a}=\alpha_{b}$ since $\beta_{a}=\alpha_{a}^{-1}, \beta_{b}=\alpha_{b}^{-1}$. Hence $(a, b) \in \mu$ if and only if $a \theta=b \theta$.

As immediate corollaries of this result, Lemma 4.1 and the two-sided analogue of Proposition 3.5, we have

Corollary 4.6. If $S$ is a type A semigroup, then $S / \mu$ is a type A semigroup.

Corollary 4.7. In the notation of Proposition 4.5, the restriction of $\theta$ to $E$ is an isomorphism onto the semilattice of idempotents of $T_{E}$ and for $a \in S$ we have $(a \theta)^{-1}(a \theta)=(a \theta)^{*}=a^{*} \theta,(a \theta)(a \theta)^{-1}=(a \theta)^{\dagger}=a^{\dagger} \theta$.

Corollary 4.8. A type A semigroup $S$ with semilattice of idempotents $E$ is fundamental if and only if it is isomorphic to a full subsemigroup of $T_{E}$.

In addition we have the following two corollaries. Recall that a semilattice $E$ is anti-uniform if $e E \approx f E$ implies $e=f$.

Corollary 4.9. A semilattice $E$ has the property that every type A semigroup having $E$ as semilattice of idempotents is a semilattice of cancellative monoids if and only if $E$ is anti-uniform.

Proof. Noting that an inverse semigroup which is a semilattice of cancellative monoids is actually a semilattice of groups, we have from (7, Chapter $V$, Theorem 5.2) that if $E$ is not anti-uniform, then $T_{E}$ is not a semilattice of cancellative monoids. On the other hand if $E$ is an anti-uniform semilattice and $S$ is a type $A$ semigroup with 
semilattice of idempotents $E$, then for each $a \in S$, we have $a^{\dagger} E \approx a^{*} E$ so that $a^{\dagger}=a^{*}$ and by Proposition 2.9, $S$ is a semilattice of cancellative monoids.

Recall that a semilattice $E$ is rigid if for each $e \in E$ there is only one automorphism of $e E$. Clearly this is the case if and only if there is at most one isomorphism between $e E$ and $f E$ for each pair $e, f \in E$.

Corollary 4.10. A semilattice $E$ has the property that $\mathscr{H}^{*}$ is a congruence on every type A semigroup having $E$ as semilattice of idempotents if and only if $E$ is rigid.

Proof. $T_{E}$ is a fundamental inverse semigroup so that on $T_{E}, \mathscr{H}^{*}=\mathscr{H}$ and $\mu=\iota$. It is well-known that $\mathscr{H}=\imath$ on $T_{E}$ if and only if $E$ is rigid. For the converse, let $E$ be a rigid semilattice and $S$ a type $A$ semigroup having $E$ as its semilattice of idempotents. Then if $a, b \in S$ and $(a, b) \in \mathscr{H}^{*}$, we have $a \dagger=b \dagger$ and $a^{*}=b^{*}$ so that $\alpha_{a}, \alpha_{b}$ have the same domain and range. Since $E$ is rigid, this gives $\alpha_{a}=\alpha_{b}$ and hence also $\beta_{a}=\beta_{b}$ so that $(a, b) \in \mu$. Thus $\mathscr{H}^{*}=\mu$ is a congruence on $S$.

\section{REFERENCES}

(1) A. H. Clifford and G. B. PRESTon, The algebraic theory of semigroups, Vol. II (Math. Surveys No. 7, Amer. Math. Soc., 1967).

(2) M. P. DOROFEEva, Hereditary and semi-hereditary monoids, Semigroup Forum 4 (1972), 301-311.

(3) J. B. FountaIN, Right PP monoids with central idempotents, Semigroup Forum 13 (1977), 229-237.

(4) J. B. Fountain, A class of right PP monoids, Quart. J. Math. Oxford to appear.

(5) T. E. HaLL, On orthodox semigroups and uniform and anti-uniform bands, J. Algebra 16 (1970), 204-217.

(6) J. M. HowIE, The maximum idempotent-separating congruence on an inverse semigroup, Proc. Edinburgh Math. Soc. (2) 14 (1964), 71-79.

(7) J. M. HowIE, An introduction to semigroup theory (Academic Press, 1976).

(8) M. KIL'P, Commutative monoids all of whose principal ideals are projective, Semigroup Forum 6 (1973), 334-339.

(9) D. B. MCALISTER, One-to-one partial right translations of a right cancellative semigroup, J. Algebra 43 (1976), 231-251.

(10) W. D. MUNN, Uniform semilattices and bisimple inverse semigroups, Quart. J. Math. Oxford (2) 17 (1966), 151-159.

(11) F. PASTIJN, A representation of a semigroup by a semigroup of matrices over a group with zero, Semigroup Forum 10 (1975), 238-249.

\section{Department of Mathematics \\ UNIVERSITY OF YORK \\ HESLINGTON}

YORK Y01 5DD 